\title{
Spin adapted implementation of EOM-CCSD for triplet excited states: Probing intersystem crossings of acetylacetone at the carbon and oxygen K-edges
}

\author{
Faber, Rasmus; Kjønstad, Eirik F.; Koch, Henrik; Coriani, Sonia
}

Published in:

Journal of Chemical Physics

Link to article, DOI:

$10.1063 / 1.5112164$

Publication date:

2019

Document Version

Publisher's PDF, also known as Version of record

Link back to DTU Orbit

Citation (APA):

Faber, R., Kjønstad, E. F., Koch, H., \& Coriani, S. (2019). Spin adapted implementation of EOM-CCSD for triplet excited states: Probing intersystem crossings of acetylacetone at the carbon and oxygen K-edges. Journal of Chemical Physics, 151(14), [144107]. https://doi.org/10.1063/1.5112164

\section{General rights}

Copyright and moral rights for the publications made accessible in the public portal are retained by the authors and/or other copyright owners and it is a condition of accessing publications that users recognise and abide by the legal requirements associated with these rights.

- Users may download and print one copy of any publication from the public portal for the purpose of private study or research.

- You may not further distribute the material or use it for any profit-making activity or commercial gain

- You may freely distribute the URL identifying the publication in the public portal 


\section{Spin adapted implementation of EOM- CCSD for triplet excited states: Probing intersystem crossings of acetylacetone at the carbon and oxygen K-edges}

Cite as: J. Chem. Phys. 151, 144107 (2019); https://doi.org/10.1063/1.5112164

Submitted: 05 June 2019 . Accepted: 11 September 2019 . Published Online: 08 October 2019

Rasmus Faber (D), Eirik F. Kjønstad (D), Henrik Koch (D), and Sonia Coriani (iD)

\section{ARTICLES YOU MAY BE INTERESTED IN}

The simulation of X-ray absorption spectra from ground and excited electronic states using core-valence separated DFT/MRCI

The Journal of Chemical Physics 151, 144104 (2019); https://doi.org/10.1063/1.5110418

An efficient spectral method for numerical time-dependent perturbation theory

The Journal of Chemical Physics 151, 144106 (2019); https://doi.org/10.1063/1.5121722

Using SCF metadynamics to extend density matrix embedding theory to excited states

The Journal of Chemical Physics 151, 034112 (2019); https://doi.org/10.1063/1.5096177

\section{Challenge us.}

What are your needs for periodic

signal detection?
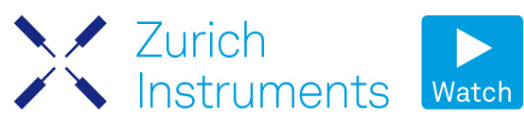

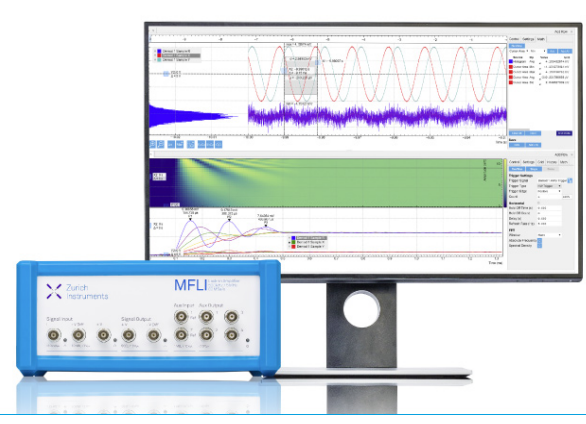

151, 144107 


\section{Spin adapted implementation of EOM-CCSD for triplet excited states: Probing intersystem crossings of acetylacetone at the carbon and oxygen K-edges}

Cite as: J. Chem. Phys. 151, 144107 (2019); doi: 10.1063/1.5112164

Submitted: 5 June 2019 Accepted: 11 September 2019 •

Published Online: 8 October 2019

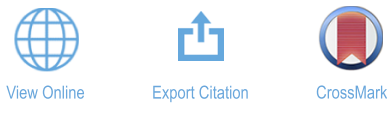

Rasmus Faber, ${ }^{7}$ (D) Eirik F. Kjønstad, ${ }^{2}$ (D) Henrik Koch, ${ }^{2,3, a)}$ (D) and Sonia Coriani ${ }^{1, b)}$ (D)

AFFILIATIONS

${ }^{1}$ DTU Chemistry, Technical University of Denmark, Kemitorvet Build. 207, DK-2800 Kongens Lyngby, Denmark

${ }^{2}$ Department of Chemistry, Norwegian University of Science and Technology, N-7491 Trondheim, Norway

${ }^{3}$ Scuola Normale Superiore, Piazza dei Cavalieri, 7, I-56126 Pisa (PI), Italy

Note: This paper is part of the JCP Special Collection on Ultrafast Spectroscopy and Diffraction from XUV to X-ray.

a) Electronic mail: henrik.koch@sns.it

b) Electronic mail: soco@kemi.dtu.dk

\section{ABSTRACT}

We present an equation of motion coupled cluster singles and doubles approach for computing transient absorption spectra from a triplet excited state. The implementation determines the left and right excitation vectors by explicitly spin-adapting the triplet excitation space. As an illustrative application, we compute transient state X-ray absorption spectra at the carbon and oxygen K-edges for the acetylacetone molecule.

Published under license by AIP Publishing. https://doi.org/10.1063/1.5112164

\section{INTRODUCTION}

Recent years have witnessed remarkable advances, in both large-facility and laboratory-based (table-top) X-ray sources, that enable a wide range of investigations with unprecedented time resolution and element specificity. ${ }^{1-4}$ Time-resolved pump-probe experimental techniques have become popular and now constitute a powerful tool to study the occurrence of fundamental processes such as internal conversion and intersystem crossing. For instance, an ultraviolet-visible (UV-vis) pump and an X-ray probe may be used to investigate the dynamics of valence excited states.

These experimental advances imply a growing need for reliable and computationally efficient tools to simulate and interpret experimental spectra. ${ }^{1,7}$ Over the last five years, a number of computational approaches have been developed to simulate transient state X-ray absorption spectra (TS-XAS) of singlet excited states. ${ }^{5,6,8-11}$ Applications include the investigation of ultrafast internal conversion in organic molecules. Particularly relevant to the present work is the recent development of coupled cluster approaches to near-edge time-resolved X-ray absorption spectroscopy (TR-XAS).

In this contribution, we broaden the spin adapted equation of motion coupled cluster singles and doubles (EOM-CCSD) approach $^{15,16}$ to the calculation of triplet transient state X-ray absorption spectra. Several popular quantum chemistry codes contain algorithms for the solution of the coupled cluster right and left eigenvalue equations for singlet excited states in both spinadapted and unrestricted formulations. For triplet excited states, current implementations are mostly unrestricted. ${ }^{17-22}$ Indeed, for a closed-shell reference, a straightforward spin-orbital implementation yields spin-pure states, and spin-adaptation is only needed to reduce the costs of the calculations. An alternative strategy is to enforce spin-symmetry in the matrix multiplications, as is done in Q-Chem ${ }^{18}$ using the libtensor library. ${ }^{23}$ Nonetheless, a fully spin-adapted implementation is, computationally, the most efficient strategy. 
To the best of our knowledge, the only spin adapted triplet CCSD implementation is the one of Hald, Hättig, and Jørgensen, ${ }^{16}$ which is limited to the solution of the right eigenvalue equation. A complete spin adapted implementation for the coupled cluster singles and approximate doubles model (CC2) using the resolution of identity approximation (RI-CC2) was presented by Hättig, Köhn, and Hald ${ }^{24}$ and generalized to intensities by Pabst and Köhn. ${ }^{2}$

Building on previous work by Hald, Hättig and Jørgensen, ${ }^{16}$ we derive and implement the CCSD left transformation for triplet excited states. Furthermore, we apply the core-valence separation in the solution of the eigenvalue equations. ${ }^{13,14}$ In this way, we obtain core excitation energies as well as spectral intensities between valence and core excited triplet states. This extends the computational methodology used in Ref. 5, which was designed for probing internal conversions by TR-XAS, to the study of intersystem crossings.

As an illustrative application, we compute the spectral signatures of the intersystem crossings in acetylacetone, for which recent experimental and theoretical (MOM-TD-DFT) results are available. ${ }^{6}$ The photoionization dynamics of acetylacetone has also been the subject of a recent experimental and theoretical effort ${ }^{26}$ using UV-pump/XUV-probe FEL (free electron laser) at Elettra. ${ }^{26}$ Finally, we predict the oxygen $\mathrm{K}$-edge spectrum, for which experimental data are currently not available.

\section{THEORY}

Coupled cluster theory employs an exponentially parametrized wavefunction,

$$
|\mathrm{CC}\rangle=\exp (T)|\mathrm{HF}\rangle,
$$

where $|\mathrm{HF}\rangle$ is the closed-shell Hartree-Fock Slater determinant and $T$ is a linear combination of excitation operators $\tau_{\mu}$,

$$
T=\sum_{\mu} t_{\mu} \tau_{\mu}
$$

with weights $t_{\mu}$ known as amplitudes. Here and in the following, $|\mu\rangle$ refers to excited configurations generated by applying the operator $\tau_{\mu}$ to $|\mathrm{HF}\rangle$. The explicit form of the operators $\tau_{\mu}$ for a closed-shell ground state can be found in Ref. 27.

The coupled cluster ground state energy and amplitudes are conventionally obtained by projection of the time-independent Schrödinger equation for $|\mathrm{CC}\rangle$ onto the reference $|\mathrm{HF}\rangle$ and the excited configurations $|\mu\rangle$. The resulting equations may be expressed as

$$
\begin{aligned}
E_{\mathrm{CC}} & =\langle\mathrm{HF}|\bar{H}| \mathrm{HF}\rangle, \\
\Omega_{\mu} & =\langle\mu|\bar{H}| \mathrm{HF}\rangle=0,
\end{aligned}
$$

where $\bar{H}=e^{-T} H e^{T}$ is known as the similarity transformed Hamiltonian. In the CCSD model, $\tau_{\mu}$ is restricted to single and double excitations relative to $|\mathrm{HF}\rangle$.

Two different approaches exist for describing excited states in coupled cluster theory. One is linear response (CCLR) theory, ${ }^{28}$ where the excitation energies are defined from the pole-structure of the linear response function and obtained by solving the nonsymmetric eigenvalue problems,

$$
\begin{aligned}
\mathbf{A R}_{n} & =\omega_{n} \mathbf{R}_{n}, \\
\mathbf{L}_{n}^{T} \mathbf{A} & =\omega_{n} \mathbf{L}_{n}^{T}, \quad n=1,2, \ldots,
\end{aligned}
$$

under the biorthonormality condition $\mathbf{L}_{m}^{T} \mathbf{R}_{n}=\delta_{m n}$. The matrix $\mathbf{A}$, defined by

$$
A_{v \mu} \equiv\left\langle v\left|\left[\bar{H}, \tau_{\mu}\right]\right| \mathrm{HF}\right\rangle,
$$

is called the coupled cluster Jacobian. The other approach is equation of motion (EOM-CC) theory, ${ }^{17,29}$ where excited states are found from a CI-like eigenvalue problem using the matrix representation of $\bar{H}$ in the basis $\{|\mathrm{HF}\rangle,|\mu\rangle\}$. Since Eq. (4) is satisfied, the two approaches give the same expression for the excitation energies.

Clearly, only the solution of the right eigenvalue equation, Eq. (5), is needed to obtain the excitation energies $\omega_{n}$. However, both right $\left(\mathbf{R}_{n}\right)$ and left $\left(\mathbf{L}_{n}\right)$ excitation vectors are needed for the computation of spectral intensities. In order to calculate transient state absorption spectra, we need the dipole transition moments between two excited states. Within the EOM-CC formalism, these can be written as ${ }^{17,30-33}$

$$
S(i \rightarrow f)=\sum_{X=x, y, z} T_{i f}^{X} T_{f i}^{X},
$$

where

$$
T_{i f}^{X}=\mathbf{L}_{i}^{T} \mathbf{A}^{X, \mathrm{EOM}} \mathbf{R}_{f}-\left(\overline{\mathbf{t}}^{T} \mathbf{R}_{f}\right)\left(\mathbf{L}_{i}^{T} \boldsymbol{\xi}^{X}\right)-\left(\mathbf{L}_{i}^{T} \mathbf{R}_{f}\right)\left(\overline{\mathbf{t}}^{T} \boldsymbol{\xi}^{X}\right) .
$$

The EOM property Jacobian $\mathbf{A}^{X, \mathrm{EOM}}$ for the operator $X$ is given by $^{30,31,34}$

$$
A_{\mu \nu}^{X, \mathrm{EOM}}=\langle\mu|\bar{X}| v\rangle-\langle\mathrm{HF}|\bar{X}| \mathrm{HF}\rangle \delta_{\mu \nu} .
$$

The vectors $\overline{\mathbf{t}}$ and $\boldsymbol{\xi}^{X}$ above are the ground-state Lagrangian multipliers and property right-hand-side vectors, respectively. Their definition can be found in Refs. 15 and 28. Note that the EOM-CC and CCLR transition moments are not identical.

In the case of triplet states, the second term in Eq. (9) vanishes as the ground state Lagrangian multipliers $\overline{\mathbf{t}}$ are necessarily singlets for a singlet ground state. Moreover, for different states $(i \neq f)$, the last term vanishes by biorthonormality. Thus, only the contraction of the $\mathbf{A}^{X, \mathrm{EOM}}$ matrix with the left and right eigenvectors needs to be evaluated.

The solution of Eq. (5) for a triplet excited state in spin-adapted CCSD has been discussed in Ref. 16. We focus here on Eq. (6), which is most conveniently solved using a direct subspace approach that only requires the ability to calculate the Jacobian left-transformation of a vector $\mathbf{L}$. The elements of the left transformed vector can be written as

$$
\rho_{\mu} \equiv\left(\mathbf{L}^{T} \mathbf{A}\right)_{\mu}=\left\langle\mathrm{HF}\left|\mathcal{L}\left[\bar{H}, \tau_{\mu}\right]\right| \mathrm{HF}\right\rangle,
$$

where

$$
\mathcal{L}=\sum_{v} \tau_{v}^{\dagger} L_{v}
$$


An analogous operator $\mathcal{R}$ can be defined when right transforming a vector $\mathbf{R}$. In the following subsections, we discuss the explicit parametrizations required to compute the transformed vector $\rho$ in the case of a triplet excitation vector.

\section{A. Parametrization of the triplet excitation space}

In order to obtain programmable expressions for Eqs. (10) and (11), we need explicit expressions for the excitation operators $\tau_{\mu}$. We follow the convention where the letters $a$ through $f$ ( $i$ through $o$ ) refer to virtual (occupied) molecular orbitals.

The construction of operators for the singlet excitation space is discussed elsewhere. ${ }^{27}$ These excitation operators are used for the ground state operators $T$ and $\Lambda=\sum_{\mu} \bar{t}_{\mu} \tau_{\mu}^{\dagger}$. To span the triplet space, we use the operators

$$
\begin{aligned}
& { }^{(3)} \tau_{a i}=a_{a \alpha}^{\dagger} a_{i \alpha}-a_{a \beta}^{\dagger} a_{i \beta} \text {, } \\
& { }^{(+)} \tau_{a i b j}=a_{a \alpha}^{\dagger} a_{i \alpha} a_{b \alpha}^{\dagger} a_{j \alpha}-a_{a \beta}^{\dagger} a_{i \beta} a_{b \beta}^{\dagger} a_{j \beta}, \\
& { }^{(-)} \tau_{a i b j}=a_{a \alpha}^{\dagger} a_{i \alpha} a_{b \beta}^{\dagger} a_{j \beta}-a_{a \beta}^{\dagger} a_{i \beta} a_{b \alpha}^{\dagger} a_{j \alpha} .
\end{aligned}
$$

To simplify the notation, we will from now on omit the superscript (3) on the triplet operator ${ }^{(3)} \tau_{a i}$, as well as in all amplitudes and terms connected to it. We emphasize once more that the operator in Eq. (13) is different from the one entering $T$ and $\Lambda$. Also, note that the above definitions differ from those of Hald, Hättig, and Jørgensen ${ }^{16}$ by a scalar factor, but it is trivial to transform between the two representations. The double excitation operators can be shown to fulfill the following symmetry relations:

$$
\begin{aligned}
& { }^{(+)} \tau_{\text {aibj }}={ }^{\left({ }^{(+)}\right.} \tau_{a j b i}=-{ }^{(+)} \tau_{\text {biaj }}={ }^{(+)} \tau_{\text {bjai }}, \\
& { }^{(-)} \tau_{\text {aibj }}={ }^{(-)} \tau_{\text {bjai }} .
\end{aligned}
$$

These relations restrict the number of linearly independent parameters. Thus, a complete parametrization of the CCSD triplet right excitation operator can be written as

$$
\begin{aligned}
\mathcal{R} & =\sum_{a i} R_{i}^{a} \tau_{a i}+\sum_{a>b, i>j}{ }^{(+)} R_{i j}^{a b}{ }^{(+)} \tau_{a i b j}+\sum_{(a i)>(b j)}{ }^{(-)} R_{i j}^{a b}{ }^{(-)} \tau_{a i b j} \\
& =\sum_{a i} R_{i}^{a} \tau_{a i}+\frac{1}{4} \sum_{a b i j}{ }^{(+)} R_{i j}^{a b}{ }^{(+)} \tau_{a i b j}+\frac{1}{2} \sum_{a b i j}{ }^{(-)} R_{i j}^{a b}{ }^{(-)} \tau_{a i b j} .
\end{aligned}
$$

Similarly, the CCSD triplet left operator can be written as

$$
\begin{aligned}
\mathcal{L} & =\frac{1}{2}\left(\sum_{a i} L_{i}^{a} \tau_{a i}^{\dagger}+\sum_{a>b, i>j}{ }^{(+)} L_{i j}^{a b(+)} \tau_{a i b j}^{\dagger}+\sum_{(a i)>(b j)}{ }^{(-)} L_{i j}^{a b(-)} \tau_{a i b j}^{\dagger}\right) \\
& =\frac{1}{2} \sum_{a i} L_{i}^{a} \tau_{a i}^{\dagger}+\frac{1}{8} \sum_{a b i j}{ }^{(+)} L_{i j}^{a b}{ }^{(+)} \tau_{a i b j}^{\dagger}+\frac{1}{4} \sum_{a b i j}{ }^{(-)} L_{i j}^{a b(-)} \tau_{a i b j}^{\dagger} .
\end{aligned}
$$

Using the above definitions, expressions for the Jacobian lefttransformed triplet vector can be derived. Since there are three kinds of operators spanning the triplet space in the CCSD case, it is convenient to also split the transformed vector into three parts as follows:

$$
\begin{aligned}
\rho_{a i} & =\left\langle\mathrm{HF}\left|\mathcal{L}\left[\bar{H}, \tau_{a i}\right]\right| \mathrm{HF}\right\rangle, \\
{ }^{(+)} \rho_{a i b j} & =\left\langle\mathrm{HF}\left|\mathcal{L}\left[\bar{H},{ }^{(+)} \tau_{a i b j}\right]\right| \mathrm{HF}\right\rangle, \\
{ }^{(-)} \rho_{a i b j} & =\left\langle\mathrm{HF}\left|\mathcal{L}\left[\bar{H},{ }^{(-)} \tau_{a i b j}\right]\right| \mathrm{HF}\right\rangle .
\end{aligned}
$$

Explicit expressions for these quantities are given in Sec. II B.

The operation count of one triplet left-transformation as implemented here is $\frac{3}{8} O^{2} N^{4}+6 O^{3} V^{3}+3 O^{4} V^{2}$, if we only count the terms that scale as the sixth power of the system size. Here, $O$ and $V$ denote the number of occupied and virtual orbitals, respectively, and $N=O+V$ is the number of basis functions. This means that the left transformation in the triplet case is about $\frac{3}{2}$ the cost of the singlet case, ${ }^{36,37}$ which is consistent with the fact that there are $\frac{3}{2}$ as many independent parameters in the triplet parametrization. Note, however, that the results presented here still rely on the implementation of the right linearly transformed vector of Hald, Hättig, and Jørgensen. ${ }^{16}$ According to the authors, ${ }^{16}$ their triplet right transformation costs $\frac{3}{8} N^{4} O^{2}+8 V^{3} O^{3}+2 V^{2} O^{4}$ vs $\frac{1}{4} N^{4} O^{2}$ $+4 V^{3} O^{3}+V^{2} O^{4}$ for a singlet right transformation, i.e., a factor of $\frac{3}{2}$ on the leading term and a factor of 2 on the remaining terms.

\section{B. Triplet left-transformation}

Following the derivation in Ref. 36, we write the singles part of the triplet left transformed vector, Eq. (20), as

$$
\rho_{a i}=\rho_{a i}^{A}+\rho_{a i}^{B}+\rho_{a i}^{C}+\rho_{a i}^{D}+\rho_{a i}^{E}+\rho_{a i}^{F}+\rho_{a i}^{G} .
$$

The various contributions are given by

$$
\begin{aligned}
\rho_{a i}^{A}= & \sum_{e m} L_{m}^{e}\left[-\hat{g}_{i m e a}+\sum_{n f} \hat{g}_{i f n a} t_{n m}^{e f}\right], \\
\rho_{a i}^{B}= & \sum_{e} L_{i}^{e} \mathcal{F}_{e a}-\sum_{m} L_{m}^{a} \mathcal{F}_{i m}, \\
\rho_{a i}^{C}= & -\sum_{e f} \hat{g}_{i f e a} Y_{e f}+\sum_{m n} \hat{g}_{i n m a} X_{m n}, \\
\rho_{a i}^{D}= & -\sum_{e} \mathcal{F}_{i e} Y_{a e}-\sum_{m} \mathcal{F}_{m a} X_{m i}, \\
\rho_{a i}^{E}= & \sum_{e f m} \tilde{L}_{i m}^{e f} \hat{g}_{e a f m}-\sum_{e m n} \tilde{L}_{m n}^{a e} \hat{g}_{i m e n}, \\
\rho_{a i}^{F}= & \sum_{n e g} \tilde{L}_{i n}^{e g} \sum_{m f}\left[\left(2 \hat{g}_{e a m f}-\hat{g}_{e f m a}\right) t_{m n}^{f g}-\hat{g}_{e a m f} t_{n m}^{f g}-\hat{g}_{m a g f} t_{m n}^{e f}\right] \\
& +\sum_{e f n} \tilde{L}_{i n}^{e f} \sum_{m o} \hat{g}_{m a o n} t_{m o}^{e f}, \\
\rho_{a i}^{G}= & -\sum_{e m n} \tilde{L}_{m n}^{a e} \sum_{o f}\left[\left(2 \hat{g}_{i m o f}-\hat{g}_{i f o m}\right) t_{n o}^{e f}-\hat{g}_{i m o f} t_{o n}^{e f}-\hat{g}_{i f o n} t_{o m}^{e f}\right] \\
& -\sum_{e m n} \tilde{L}_{m n}^{a e} \sum_{f g} \hat{g}_{i f e g} t_{m n}^{f g},
\end{aligned}
$$

where $\tilde{L}_{m n}^{e f}={ }^{(+)} L_{m n}^{e f}+{ }^{(-)} L_{m n}^{e f}$ and $\hat{g}_{p q r s}$ are the $T_{1}$ transformed twoelectron repulsion integrals.

In the above equations, we have introduced a number of intermediates. The one-particle intermediates are the same as those in the 
singlet case, ${ }^{36}$

$$
\begin{aligned}
& \mathcal{F}_{e a}=F_{e a}+\sum_{m f}\left(2 \hat{g}_{\text {eamf }}-\hat{g}_{e f m a}\right) t_{m}^{f}-\sum_{f m n}\left(2 \hat{g}_{m a n f}-\hat{g}_{m f n a}\right) t_{m n}^{e f}, \\
& \mathcal{F}_{i m}=F_{i m}+\sum_{e n}\left(2 \hat{g}_{i m n e}-\hat{g}_{i e n m}\right) t_{n}^{e}+\sum_{e f n}\left(2 \hat{g}_{i e n f}-\hat{g}_{i f n e}\right) t_{m n}^{e f}, \\
& \mathcal{F}_{j b}=\sum_{e m}\left(2 \hat{g}_{m e j b}-\hat{g}_{m b j e}\right) t_{m}^{e},
\end{aligned}
$$

that can also be rewritten as

$$
\begin{aligned}
& \mathcal{F}_{e a}=\hat{h}_{e a}+\sum_{m}\left(2 \hat{g}_{\text {eamm }}-\hat{g}_{e m m a}\right)-\sum_{f m n}\left(2 \hat{g}_{m a n f}-\hat{g}_{m f n a}\right) t_{m n}^{e f}, \\
& \mathcal{F}_{i m}=\hat{h}_{i m}+\sum_{n}\left(2 \hat{g}_{i m n n}-\hat{g}_{i m n m}\right)+\sum_{e f n}\left(2 \hat{g}_{i e n f}-\hat{g}_{i f n e}\right) t_{m n}^{e f}, \\
& \mathcal{F}_{j b}=\hat{h}_{j b}+\sum_{m}\left(2 \hat{g}_{j b m m}-\hat{g}_{j m m b}\right),
\end{aligned}
$$

where $\hat{h}_{p q}$ are matrix elements of the $T_{1}$ transformed one-electron Hamiltonian. The $X$ and $Y$ intermediates are also very similar to their singlet counterparts,

$$
\begin{aligned}
& X_{m i}=\sum_{e f n} \tilde{L}_{i n}^{e f} t_{m n}^{e f}, \\
& Y_{a e}=\sum_{f m n} \tilde{L}_{m n}^{a f} t_{m n}^{e f} .
\end{aligned}
$$

To handle the doubles part, it is convenient to introduce an operator that generates permutations among sets of indices,

$$
P_{p q, r s}^{(-)} a_{p q r s}=a_{p q r s}-a_{r s p q} .
$$

Using this operator, the contributions to the doubles part of the linearly transformed vector, Eqs. (21) and (22), can be written as

$$
\begin{aligned}
{ }^{(+)} \rho_{a i b j}= & { }^{(+)} \rho_{a i b j}^{A}+{ }^{(+)} \rho_{a i b j}^{B}+{ }^{(+)} \rho_{a i b j}^{E}+P_{a, b}^{(-)} P_{i, j}^{(-)} \\
& \times\left[\rho_{a i b j}^{C}+\rho_{a i b j}^{D}+\rho_{a i b j}^{F}+\frac{1}{2}{ }^{(+)} \rho_{a i b j}^{G}+\rho_{a i b j}^{H}\right], \\
{ }^{(-)} \rho_{a i b j}= & { }^{(-)} \rho_{a i b j}^{A}+{ }^{(-)} \rho_{a i b j}^{B}+{ }^{(-)} \rho_{a i b j}^{E} \\
& +P_{a i b j}^{(-)}\left[\rho_{a i b j}^{C}+{ }^{(-)} \rho_{a i b j}^{C}+\rho_{a i b j}^{D}+\rho_{a i b j}^{F}+{ }^{(-)} \rho_{a i b j}^{G}+\rho_{a i b j}^{H}\right],
\end{aligned}
$$

where

$$
\begin{aligned}
{ }^{( \pm)} \rho_{a i b j}^{A} & =\sum_{m n}{ }^{( \pm)} L_{m n}^{a b}\left[\hat{g}_{i m j n}+\sum_{e f} \hat{g}_{i e j f} t_{m n}^{e f}\right], \\
{ }^{( \pm)} \rho_{a i b j}^{B} & =\sum_{e f}{ }^{( \pm)} L_{i j}^{e f} \hat{g}_{e a f b}, \\
{ }^{( \pm)} \rho_{a i b j}^{E} & =\sum_{e f m n}{ }^{( \pm)} L_{i j}^{e f} \hat{g}_{m a n b} t_{m n}^{e f}, \\
\rho_{a i b j}^{C} & =-\frac{1}{2} \sum_{e m} \tilde{L}_{m j}^{e b}\left[\hat{g}_{e a i m}-\sum_{f m} \hat{g}_{\text {naif }} t_{n m}^{e f}\right],
\end{aligned}
$$

$$
\begin{aligned}
\rho_{a i b j}^{D}= & \frac{1}{2} \sum_{e m} \tilde{L}_{i m}^{a e}\left[\left(2 \hat{g}_{e m j b}-\hat{g}_{\text {ebjm }}\right)\right. \\
& \left.+\sum_{f n}\left(2 \hat{g}_{n f j b}-\hat{g}_{n b j f}\right)\left(2 t_{m n}^{e f}-t_{n m}^{e f}\right)\right], \\
{ }^{(-)} \rho_{a i b j}^{C}= & -\sum_{m e}{ }^{(-)} L_{m j}^{a e}\left[\hat{g}_{e b i m}-\sum_{f n} \hat{g}_{i f n b} t_{n m}^{e f}\right], \\
\rho_{a i b j}^{F}= & \sum_{m} \hat{g}_{i a m b} X_{m j}-\sum_{e} \hat{g}_{i e j b} Y_{a e}, \\
{ }_{( \pm)} \rho_{a i b j}^{G}= & \sum_{e}{ }^{( \pm)} L_{i j}^{a e} \mathcal{F}_{e b}-\sum_{m}{ }^{( \pm)} L_{i m}^{a b} \mathcal{F}_{j m}, \\
\rho_{a i b j}^{H}= & L_{i}^{a} \mathcal{F}_{j b}+\sum_{e} L_{i}^{e} \hat{g}_{e a j b}-\sum_{m} L_{m}^{a} \hat{g}_{i m j b} .
\end{aligned}
$$

We refer the interested reader to the Appendix for additional details on our implementation in Dalton. ${ }^{20}$

\section{INTERSYSTEM CROSSINGS IN ACETYLACETONE}

We have used the methodology described in the previous sections to compute spectral signatures of intersystem crossings (ISC) in acetylacetone at the carbon and oxygen K-edges. Spectral signatures of internal conversion (IC) processes have also been computed for completeness.

In a time-resolved UV-pump/X-ray-probe experiment recently carried out on acetylacetone at the carbon K-edge by Bhattacherjee et al. ${ }^{6}$ using table-top instrumentation, the system is initially brought into a valence excited state by absorption of UV radiation (see scheme (a) in Fig. 1 for a representation of the process). The probe frequency is tuned to the $S_{2}$ electronic singlet excited state of $\pi \pi^{\star}$ character. From $S_{2}$, the system can undergo internal conversion into another singlet $S_{1}$ excited state of $n \pi^{\star}$ character and/or undergo intersystem crossing into an excited state $T_{1}$ of triplet spin-symmetry. The system can be probed, at different time delays, with an X-ray pulse. This pulse excites specific inner shell electrons of the valence excited states that are populated at a given time delay, depending on the probed K-edge region.

Our calculations focus on the spectral signatures that are due to the process illustrated by scheme (b) in Fig. 1. They are new,

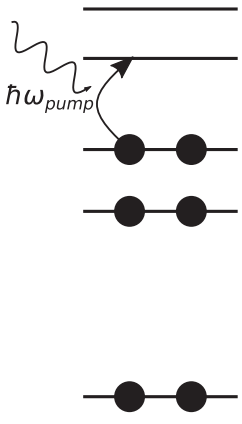

(a)

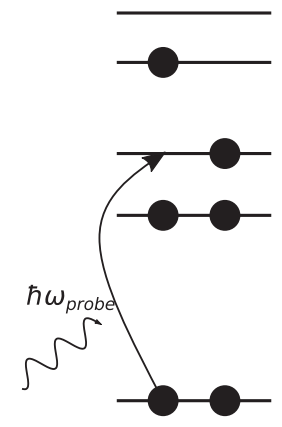

(b)

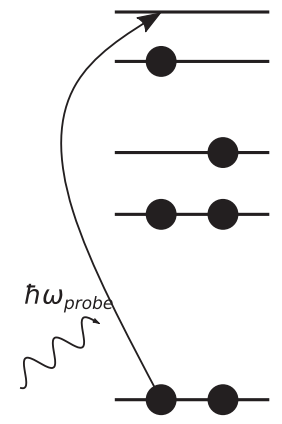

(c)
FIG. 1. Schematic overview of the processes involved in the TR-XAS pump-probe experiment. 
well defined signatures emerging at energies below the first core excitation of the ground state. As seen in Fig. 1, they correspond to the process by which one $1 \mathrm{~s}$ electron fills the vacancy in the valence orbitals (SOMOs) of the valence excited state, generated either by the pump excitation or by IC/ISC. The final state is clearly single-excitation dominated and therefore well described by CCSD. From the experimental point of view, the core-to-SOMO peaks are a preferred choice when tracking excited-state dynamics, since they unambiguously originate from the valence-excited states that are probed. $5,11,12,38$ Other mechanisms can of course also occur; see, e.g., scheme (c) in Fig. 1.

Here, we compute the spectra as transitions between EOM states generated from the ground state $S_{0}$. This implies requirements on the excitation manifold. For instance, single excitations of valence excited states can be double excitations relative to $S_{0}$ [Fig. 1(c)] such that triple excitations might be important. Therefore, some spectral features may be missing or be located at too high energy. A possible strategy to partly overcome this problem is to compute the spectra as linear absorption spectra of a highspin reference mimicking the character of the valence excited state, as explored for instance in Refs. 38 and 39. Our current implementation in Dalton does not allow us to carry out these types of calculations. We note nonetheless that spectral signatures from these other processes are expected to overlap with the ground state spectrum.

Starting from the CASPT2 geometries in Ref. 26, we reoptimized the acetylacetone molecular structures of the ground state $S_{0}$, the two lowest singlet states, $S_{1}\left(n \pi^{*}\right)$ and $S_{2}\left(\pi \pi^{*}\right)$, and the lowest triplet excited state $T_{1}\left(\pi \pi^{*}\right)$ using QChem ${ }^{18}$ and (EOM-)CCSD/aug-cc-pVDZ. In each case, the structure found was the enol tautomer.

The bond lengths that changed significantly between the various states are given in Table I. The full set of coordinates for each geometry is given in the supplementary material file.

All excitation energies and oscillator strengths were computed with a development version of Dalton. ${ }^{20}$ To generate the spectra, we used Lorentzian broadening with a half width at half maximum (HWHM) of $0.2 \mathrm{eV}$. The oscillator strengths $f(i \rightarrow f)$ between two states $i$ and $f$ are given by the expression

$$
f(i \rightarrow f)=\frac{2}{3}\left(\omega_{f}-\omega_{i}\right) S(i \rightarrow f) .
$$

TABLE I. Atom labeling and selected bond-distances $(\AA)$ for the relaxed geometry of each state.

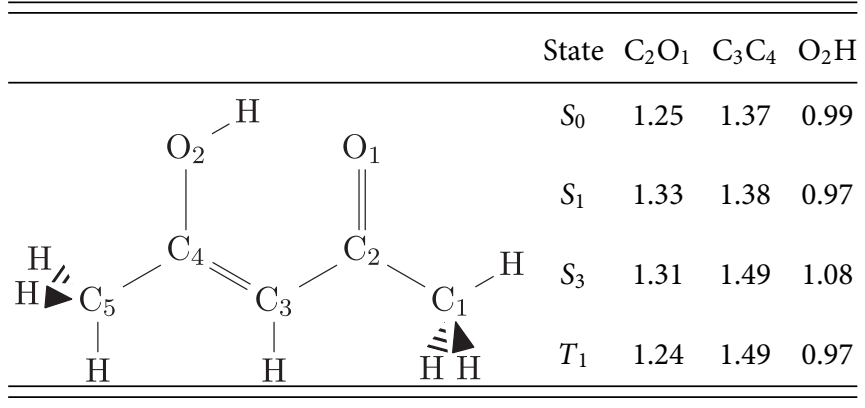

In the Dalton calculations, we used the $6-311++\mathrm{G}^{* *}$ basis set. This basis set has been shown to yield results for X-ray absorption and ionization of quality almost comparable to the aug-ccpVTZ basis set, but with reduced computational cost, both in coupled cluster ${ }^{11,40}$ and algebraic diagrammatic construction ${ }^{41,42}$ theories.

We characterized the valence excited states by means of natural transition orbitals (NTOs). These orbitals are generated from a singular value decomposition of the single-excitation part of the right excitation vector. ${ }^{43}$ Since all valence excited states are dominated by one singular value, we only consider one pair of NTOs for each state. The NTOs were visualized using MOLDEN. ${ }^{44}$ In Fig. 2, we show the NTOs obtained for the valence excited states at optimized ground and excited state geometries. We assigned the excited states by inspecting the NTOs. The energy profiles at the ground state geometry are in line with the profiles in Ref. 26.

Some notable features are evident in Fig. 2. At the minimum of the $S_{2}\left(\pi \pi^{*}\right)$ state, the $S_{1}\left(n \pi^{*}\right)$ and $S_{2}\left(\pi \pi^{*}\right)$ states are nearly degenerate $(0.06 \mathrm{eV})$, with reversed energy ordering compared to the ground state geometry. This suggests the presence of an $S_{1}\left(n \pi^{*}\right) / S_{2}\left(\pi \pi^{*}\right)$ conical intersection close to the $S_{2}\left(\pi \pi^{*}\right)$ minimum, facilitating an internal conversion into $S_{1}\left(n \pi^{*}\right)$ after initial excitation into the bright $S_{2}\left(\pi \pi^{*}\right)$ state. This is consistent with the experimental study of Bhattacherjee et al., ${ }^{6}$ where $S_{2}\left(\pi \pi^{*}\right) / S_{1}\left(n \pi^{*}\right)$ internal conversion, followed by an $S_{1}\left(n \pi^{*}\right) / T_{1}\left(\pi \pi^{*}\right)$ intersystem crossing, was deemed the most favored pathway. We should point out that the triplet $T_{2}\left(n \pi^{*}\right)$ state may act as a doorway state. In a study by Squibb et al., ${ }^{26}$ dynamics simulations (CASSCF) indicate a $S_{2} \rightarrow S_{1} \rightarrow T_{2} \rightarrow T_{1}$ pathway where the population of the $T_{2}$ state never exceeds $10 \%$. The conical intersections involved in these pathways may be investigated using similarity constrained coupled cluster theory, providing an accurate account of dynamical correlation. ${ }^{45,46}$

At the ground state geometry, the hole NTO of the $S_{1}\left(n \pi^{*}\right)$ state has a significant contribution on $\mathrm{O}_{1}$ (carbonyl group; see Table I) consistent with the interpretation of this state involving the excitation out of a nonbonding orbital on this $\mathrm{O}$. This could also explain why the spectral signature of the $S_{1}\left(n \pi^{*}\right)$ state is weak in the experimental carbon K-edge spectrum, which has rendered the dynamical evolution of this state unclear. ${ }^{6}$ It also suggests that an oxygen Kedge spectrum would be able to monitor the dynamical evolution of the $S_{1}\left(n \pi^{*}\right)$ state. $^{5}$

After a few picoseconds, two bands appear in the time-resolved spectrum, at 281.4 and $283.8 \mathrm{eV}$, that are believed to stem from the $T_{1}\left(\pi \pi^{*}\right)$ state. ${ }^{6}$ In Fig. 3, we show the computed CVS-CCSD/6$311++\mathrm{G}^{* *}$ ground and excited state carbon K-edge spectra. In Fig. 3(a), the spectra were computed at the ground state geometry. In Fig. 3(b), we show the computed transient state spectra for relaxed excited state geometries. Superimposed on the computed spectra are shaded areas in correspondence to the experimental $T_{1}\left(\pi \pi^{*}\right)$ bands, whose widths we have chosen to reflect experimental uncertainty in Ref. 6. Note also that the transient state spectra have not been scaled to account for the percentage of excited molecules (a standard downscaling is $10 \%)$. To align the ground state spectrum with experiment, we have applied an overall shift of $-1.4 \mathrm{eV}$. The computed ground state spectrum coincides with experiment ${ }^{6}$ for the second and third peaks, but underestimates the separation of the first two peaks. 


\begin{tabular}{|c|c|c|c|}
\hline Geom. & State & Hole & Particle \\
\hline \multirow{8}{*}{$\left(S_{0}\right)_{\min }$} & $S_{1}$ & & \\
\hline & $\omega=4.42 \mathrm{eV}$ & & \\
\hline & $f=0.0006$ & & \\
\hline & Character $n \pi^{*}$ & & \\
\hline & $S_{2}$ & & \\
\hline & $\omega=5.27 \mathrm{eV}$ & & \\
\hline & $f=0.3209$ & & \\
\hline & Character $\pi \pi^{*}$ & & \\
\hline
\end{tabular}

$T_{1}$

$\omega=3.72 \mathrm{eV}$

$f=0.0000$

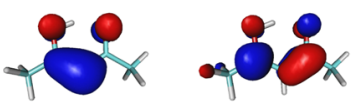

Character $\pi \pi^{*}$

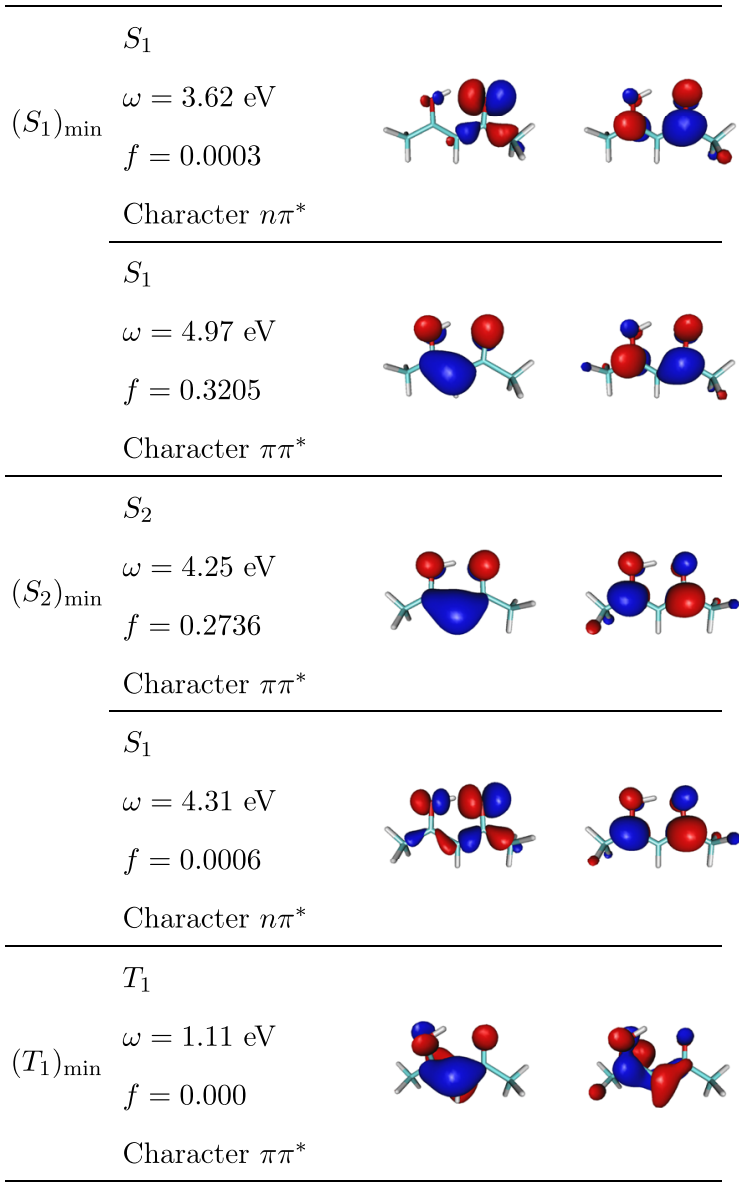

FIG. 2. CCSD/6-311++G** excitation energies $\omega(\mathrm{eV})$, oscillator strengths $f$ from the ground state, and NTOs of the singlet and triplet valence excited states considered here, computed at optimized ground and excited state geometries.

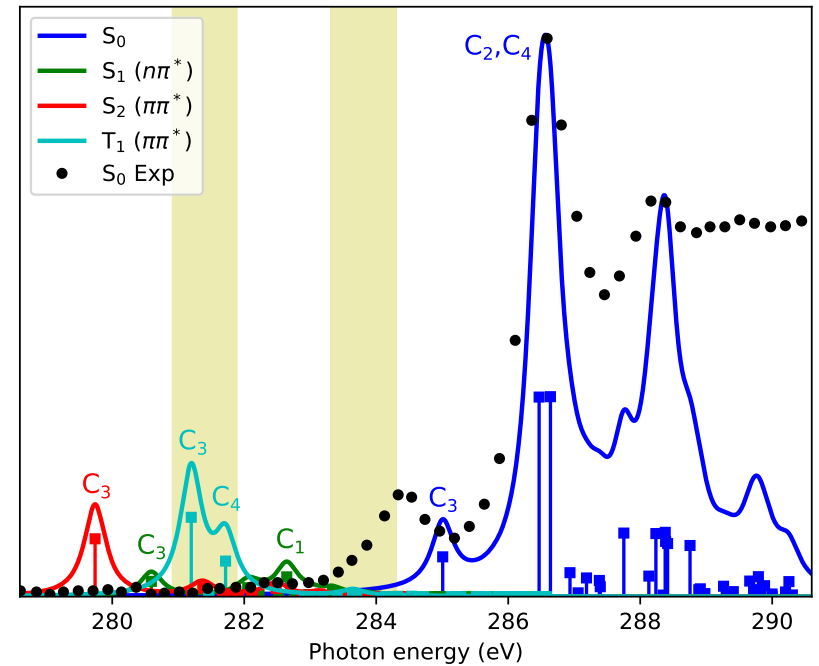

(a)

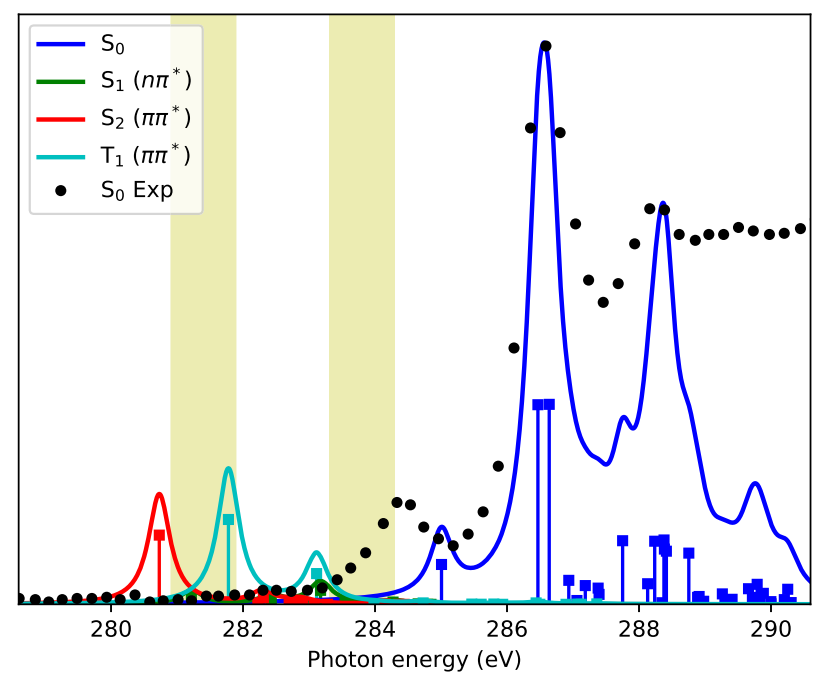

(b)

FIG. 3. CVS-CCSD/6-311++G** ground and excited state XAS spectra of acetylacetone at the carbon K-edge. Computed spectra were shifted by $-1.4 \mathrm{eV}$ to align the main peak of the computed $S_{0}$ spectrum with experiment. Labels indicate on which carbon atom the K-edge excitation takes place, numbered according to Table I. Yellow columns mark the location of experimental features interpreted as originating from the $T_{1}$ state by Bhattacherjee et al., ${ }^{6}$ emerging approximately 2 ps after the pump excitation. (a) All spectra computed at the ground state geometry. (b) Each spectrum computed at the stationary point geometry of the state it refers to.

In all computed spectra in Fig. 3, the most intense spectral features in the region $280-284 \mathrm{eV}$ are due to the two $\pi \pi^{*}$ excited states $\left(S_{2}\right.$ and $\left.T_{1}\right)$. The intensity of the $S_{1}\left(n \pi^{*}\right)$ state is weak in comparison. Measurements of TS-XAS at the carbon K-edge are therefore not suitable to monitor the decay of the $S_{1}\left(n \pi^{*}\right)$ state. The signature of the $S_{2}\left(\pi \pi^{*}\right)$ state, located close to $280 \mathrm{eV}$ in the 
computed spectra, is not visible in the experimental TS-XAS, supporting the conclusion that an internal conversion to the $S_{1}\left(n \pi^{*}\right)$ state occurs rapidly after initial excitation into the $S_{2}\left(\pi \pi^{*}\right)$ state. On the other hand, the computed spectra show two peaks associated with the triplet $T_{1}\left(\pi \pi^{*}\right)$, at 281.3 and $281.7 \mathrm{eVs}$ (unrelaxed) and 281.8 and $283.1 \mathrm{eVs}$ (relaxed), roughly reproducing the experimental bands at 281.4 and $283.8 \mathrm{eVs}$. ${ }^{6}$ Improved agreement with experiment may be obtained by accounting for triple

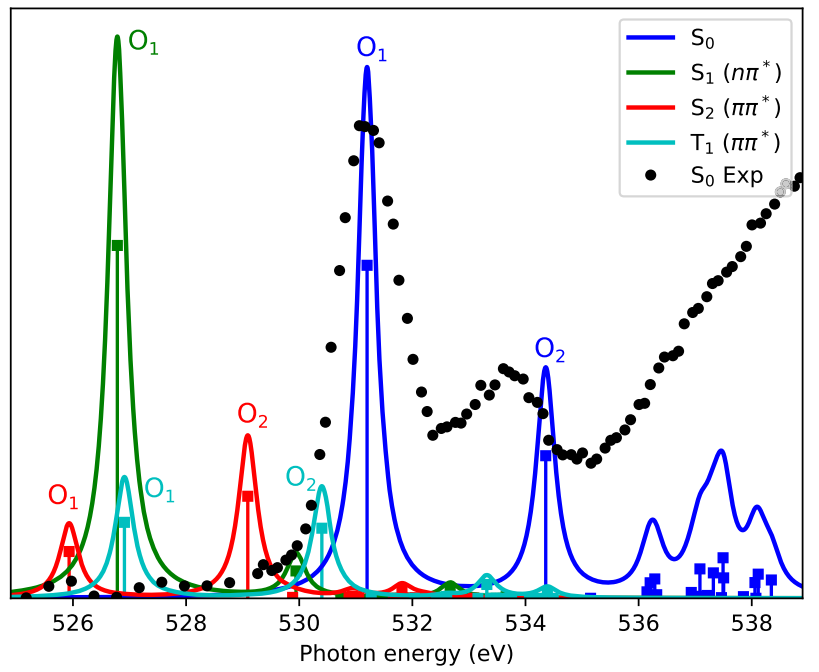

(a)

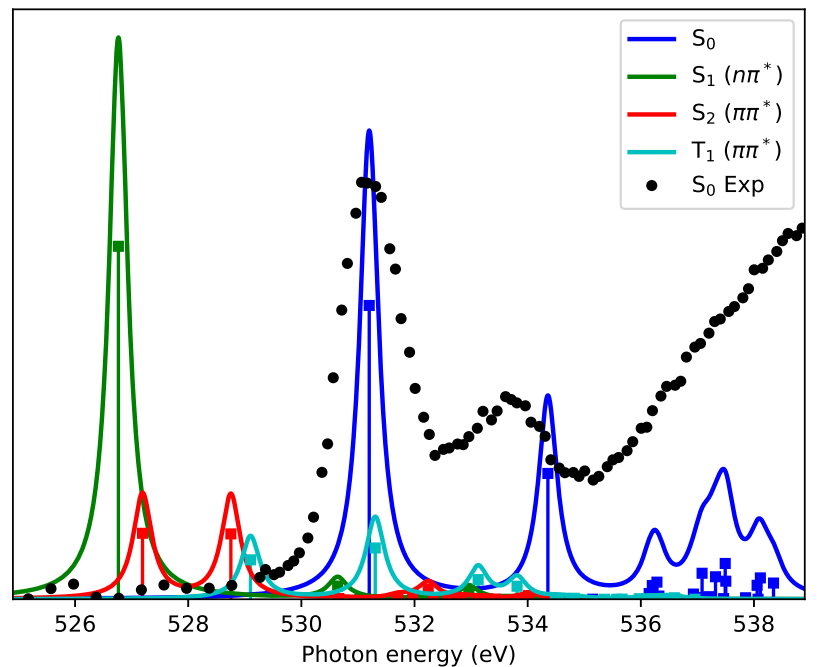

(b)

FIG. 4. CVS-CCSD/6-311++G** ground and excited state XAS spectra of acetylacetone at the oxygen K-edge. The experimental spectrum is from Refs. 48 and 49. Computed spectra were shifted by $-2.1 \mathrm{eV}$ to align the main peak of the computed ground state spectrum with experiment. Labels indicate on which oxygen atom the K-edge excitation takes place, numbered according to Table I. (a) All spectra computed at the ground state geometry. (b) Each spectrum computed at the stationary point geometry of the state it refers to. excitations, for instance, using the coupled cluster singles doubles and perturbative triples (CC3) model. ${ }^{5}$

Finally, we show in Fig. 4 the computed oxygen K-edge spectra at the Franck-Condon and relaxed geometries. A notable difference from the carbon $\mathrm{K}$-edge spectra is the strong spectral signature of core excitations from the $S_{1}\left(n \pi^{*}\right)$ state. We may therefore conclude, with some confidence, that oxygen K-edge TS-XAS experiments would be ideally suitable to probe the internal conversion from $S_{2}\left(\pi \pi^{*}\right)$ to $S_{1}\left(n \pi^{*}\right)$. Recall that the strong signature from the $S_{1}\left(n \pi^{*}\right)$ state may be understood from the orbital hole contributions at the $\mathrm{O}_{1}$ oxygen.

\section{CONCLUDING REMARKS}

We have presented a spin-adapted implementation of the EOM-CCSD left transformations that allows for the calculation of the spectral intensity between excited states. Triplet core excited states were calculated using the core-valence separation, which allow the calculation of spectral intensities between valence and core excited triplet states.

We applied the implementation to compute transient state core absorption spectra in the acetylacetone molecule. The $S_{1}, S_{2}$, and $T_{1}$ states are considered central to the decay process of this molecule following UV excitation into the $S_{2}$ state. At the carbon K-edge, the computed signature of the triplet state was found to be in qualitative agreement with time-resolved experimental spectra. Theoretical oxygen K-edge spectra, for which experimental data are currently unavailable, were also computed. They indicate that oxygen K-edge TR-XAS measurements may monitor the formation by internal conversion, and decay, of the $S_{1}$ state, which is not captured by the carbon K-edge spectra.

\section{SUPPLEMENTARY MATERIAL}

See the supplementary material for coordinates of the optimized structures and for tables of computed excitation energies and oscillator strengths.

\section{ACKNOWLEDGMENTS}

R.F. and S.C. acknowledge financial support from DTU Chemistry and from the Independent Research Fund Denmark-DFFResearch Project 2, Grant No. 7014-00258B. H.K. acknowledges support from the Otto Mønsted Fund, Denmark, for a visiting professorship at DTU Chemistry. Support from the European Union's Horizon 2020 research and innovation programme under the Marie Skłodowska-Curie Grant Agreement No. 765739, "COSINE-European Training Network on COmputational Spectroscopy In Natural sciences and Engineering" is also acknowledged. H.K. and E.F.K. acknowledge the Norwegian Research Council grants: CCGPU (Grant No. 263110) and TheoLight (Grant No. 275506). E.F.K. acknowledges Fondet til Professor Leif Tronstads minne.

\section{APPENDIX: IMPLEMENTATION}

In the following, we summarize how Eqs. (23)-(50) have been implemented. Here, Greek indices $\alpha$ through $\delta$ will refer to atomic orbitals. 


\section{Singles contributions}

The calculation of the $\mathrm{B}$ and $\mathrm{D}$ contributions is straightforward and equivalent to what has been done in the singlet case. ${ }^{36}$ The A and $\mathrm{C}$ contributions are calculated as

$$
\rho_{a i}^{A}+\rho_{a i}^{C}=-\sum_{\alpha \beta} g_{i \beta \alpha a} \mathcal{D}_{\alpha \beta}=-\sum_{\alpha \beta \gamma \delta} C_{\gamma i} C_{\delta a} g_{\gamma \beta \alpha \delta} \mathcal{D}_{\alpha \beta},
$$

where $C_{\alpha p}$ are molecular orbital coefficients. Introducing the lambda matrices,

$$
\begin{aligned}
\Lambda_{\gamma i}^{h} & =C_{\gamma i}+\sum_{e} C_{\gamma e} t_{i}^{e}, \\
\Lambda_{\gamma a}^{p} & =C_{\gamma a}-\sum_{m} C_{\gamma m} t_{m}^{a},
\end{aligned}
$$

allows us to write the $\mathcal{D}$ intermediate as

$$
\begin{aligned}
\mathcal{D}_{\alpha \beta}= & \sum_{m e} L_{m}^{e} \Lambda_{\beta m}^{h} \Lambda_{\alpha e}^{p}-\sum_{m n e f} t_{n m}^{e f} L_{m}^{e} C_{\beta f} C_{\alpha n} \\
& +\sum_{e f} Y_{e f} \Lambda_{\alpha e}^{p} C_{\beta f}-\sum_{m n} X_{m n} C_{\alpha m} \Lambda_{\beta n}^{h} .
\end{aligned}
$$

Using the partially back-transformed vector

$$
\tilde{L}_{i m}^{e \delta}=\sum_{f} \tilde{L}_{i m}^{e f} \Lambda_{\delta f}^{p}
$$

the first part of the E term can be written as

$$
\rho_{a i}^{E 1}=\sum_{e m \delta} \tilde{L}_{i m}^{e \delta} \hat{g}_{e a \delta m}
$$

The last part of the $\mathrm{E}$ and $\mathrm{G}$ terms is calculated using the BF term from the integral code, as it is done in the singlet case. ${ }^{36}$ The first part of the F term can be rewritten as

$$
\begin{aligned}
\rho_{a i}^{F 1}= & \sum_{n e g} \tilde{L}_{i n}^{e g} \sum_{m f}\left[\frac{1}{2}\left(2 \hat{g}_{\text {eamf }}-\hat{g}_{\text {efma }}\right)\left(2 t_{m n}^{f g}-t_{n m}^{f g}\right)\right. \\
& \left.-\frac{1}{2} \hat{g}_{\text {maef }} t_{n m}^{f g}-\hat{g}_{\text {magf }} t_{m n}^{e f}\right] .
\end{aligned}
$$

This expression can then be simplified by introducing the two intermediates,

$$
\begin{aligned}
P_{e i m f} & =\sum_{n g} \tilde{L}_{i n}^{e g}\left(2 t_{m n}^{f g}-t_{n m}^{f g}\right), \\
Q_{e i m f} & =-\sum_{n g}\left(\tilde{L}_{n i}^{g e}-2^{(-)} L_{i n}^{g e}\right) t_{m n}^{g f},
\end{aligned}
$$

giving

$$
\begin{aligned}
\rho_{a i}^{F 1} & =\frac{1}{2} \sum_{\text {emf }} P_{\text {eimf }}\left(2 \hat{g}_{\text {eamf }}-\hat{g}_{\text {efma }}\right)-\frac{1}{2} \sum_{\text {emf }} Q_{\text {eimf }} \hat{g}_{\text {maef }} \\
& =\sum_{\text {emf }} P_{\text {eimf }} \hat{g}_{\text {eamf }}-\frac{1}{2} \sum_{\text {emf }}\left(P_{\text {eimf }}+Q_{\text {eimf }}\right) \hat{g}_{\text {maef }} .
\end{aligned}
$$

The second part of the F term can be written as

$$
\rho_{a i}^{F 2}=\sum_{m n o} M_{\text {mion }} \hat{g}_{\text {maon }}
$$

where the $M$ intermediate is defined as

$$
M_{\text {mion }}=\sum_{e f} \tilde{L}_{i n}^{e f} t_{m o}^{e f}
$$

The first part of the G term is calculated using the same approach as the first part of the F term. That is,

$$
\rho_{a i}^{G 1}=\sum_{m o f} P_{a m o f} \hat{g}_{i m o f}-\frac{1}{2} \sum_{m o f}\left(P_{a m o f}+Q_{a m o f}^{\prime}\right) \hat{g}_{i f o m},
$$

where

$$
Q_{\text {eimf }}^{\prime}=-\sum_{n g}\left(\tilde{L}_{n i}^{g e}+2^{(-)} L_{i n}^{g e}\right) t_{m n}^{g f} .
$$

\section{Doubles contributions}

The operations in terms $\mathrm{A}, \mathrm{B}$, and $\mathrm{E}$ preserve the columnpermutation symmetry of the given $L$. This means that

$$
P_{a, b}^{(-)} P_{i, j}^{(-)(-)} \rho_{a i b j}^{X}=P_{a i, b j}^{(+)} \rho_{a i b j}^{X}=0,
$$

if $X$ is one of $A, B$, or $E$. If we define the nonsymmetric version of these three terms,

$$
\begin{aligned}
& \rho_{a i b j}^{A}=\sum_{m n} \tilde{L}_{m n}^{a b}\left[\hat{g}_{i m j n}+\sum_{e f} \hat{g}_{i e j f} t_{m n}^{e f}\right], \\
& \rho_{a i b j}^{B}=\sum_{e f} \tilde{L}_{i j}^{e f} \hat{g}_{e a f b}, \\
& \rho_{a i b j}^{E}=\sum_{e f m n} \tilde{L}_{i j}^{e f} \hat{g}_{m a n b} t_{m n}^{e f},
\end{aligned}
$$

the + and - contributions, Eqs. (42)-(44), can easily be recovered,

$$
\begin{aligned}
{ }^{(+)} \rho_{a i b j}^{X} & =\frac{1}{4} P_{a, b}^{(-)} P_{i, j}^{(-)} \rho_{a i b j}^{X}, \\
{ }^{(-)} \rho_{a i b j}^{X} & =\frac{1}{2} P_{a i, b}^{(-)} \rho_{a i b j}^{X} .
\end{aligned}
$$

Thus, it is advantageous to first calculate a nonsymmetric, general contribution

$$
\begin{aligned}
\rho_{a i b j}= & \frac{1}{8}\left(2+P_{a i, b j}^{(-)}\right)\left[\rho_{a i b j}^{A}+\rho_{a i b j}^{B}+\rho_{a i b j}^{E}\right]+\rho_{a i b j}^{C}+\rho_{a i b j}^{D} \\
& +\rho_{a i b j}^{F}+\rho_{a i b j}^{H},
\end{aligned}
$$

where $\frac{1}{8}\left(2+P_{a i, b j}^{(-)}\right)$takes care of the different prefactors of Eqs. (A19) and (A20). Equations (40) and (41) can then be written in a more compact way as

$$
\begin{aligned}
& { }^{(+)} \rho_{a i b j}=P_{a, b}^{(-)} P_{i, j}^{(-)}\left[\rho_{a i b j}+\frac{1}{2}{ }^{(+)} \rho_{a i b j}^{G}\right], \\
& { }^{(-)} \rho_{a i b j}=P_{a i, b j}^{(-)}\left[\rho_{a i b j}+{ }^{(-)} \rho_{a i b j}^{C}+{ }^{C} \rho_{a i b j}^{G}\right],
\end{aligned}
$$

leaving only three terms that are different between the $(+)$ and $(-)$ transformed vectors. 
The term in square brackets in Eq. (A16) is the $\Gamma$ intermediate of Refs. 15 and 50 and is stored on the disk. The B term is calculated by using the back-transformed left vector,

$$
\rho_{a i b j}^{B}=\sum_{\gamma \delta}\left[\sum_{e f} \Lambda_{\gamma e}^{p} \Lambda_{\delta f}^{p} \tilde{L}_{i j}^{e f}\right] g_{\gamma a \delta b}
$$

Rewriting the second part of the $\mathrm{H}$ term using the intermediate

$$
\breve{L}_{i j}^{\gamma \delta}=\sum_{e} L_{i}^{e} \Lambda_{\gamma e}^{p} C_{\delta j}
$$

the term and its contribution to the $(+)$ and $(-)$ doubles become

$$
\begin{gathered}
\rho_{a i b j}^{H 2}=\sum_{e} L_{i}^{e} \hat{g}_{e a j b}=\sum_{\gamma \delta} \breve{L}_{i j}^{\gamma \delta} \hat{g}_{\gamma a \delta b}, \\
{ }^{(+)} \rho_{a i b j}^{H 2}=P_{a, b}^{(-)} P_{i, j}^{(-)} \rho_{a i b j}^{H 2}=\left[\breve{L}_{i j}^{\gamma \delta}-\breve{L}_{j i}^{\gamma \delta}-\breve{L}_{i j}^{\delta \gamma}+\breve{L}_{j i}^{\delta \gamma}\right] g_{\gamma a \delta b}, \\
{ }^{(-)} \rho_{a i b j}^{H 2}=P_{a i, b}^{(-)} \rho_{a i b j}^{H 2}=\left[\breve{L}_{i j}^{\gamma \delta}-\breve{L}_{j i}^{\delta \gamma}\right] g_{\gamma a \delta b} .
\end{gathered}
$$

Written in this way, ${ }^{(+)} \rho_{\text {aibj }}^{H 2}$ and ${ }^{(-)} \rho_{\text {aibj }}^{H 2}$ follow the symmetry conditions, Eq. (A15), and we then calculate the sum ${ }^{(+)} \rho_{a i b j}{ }^{H 2}{ }^{(-)} \rho_{a i b j}^{H 2}$. In addition, the sum and contraction with $g_{\gamma a \delta b}$ is exactly equivalent to that in Eq. (A24), so we can calculate the sum of the B and H2 terms together,

$$
\rho_{a i b j}^{B H}=\sum_{\gamma \delta}\left[\sum_{e f} \Lambda_{\gamma e}^{p} \Lambda_{\delta f}^{p} \tilde{L}_{i j}^{e f}+2 \breve{L}_{i j}^{\gamma \delta}-\breve{L}_{j i}^{\gamma \delta}-\breve{L}_{i j}^{\delta \gamma}\right] g_{\gamma a \delta b},
$$

where

$$
\rho_{a i b j}^{B H}=\rho_{a i b j}^{B}+{ }^{(+)} \rho_{a i b j}^{H 2}+{ }^{(-)} \rho_{a i b j}^{H 2} .
$$

This term is, at $O^{2} N^{4}$, computationally the most expensive part of the left transformation. The cost is reduced using the symmetry of the integrals and vector quantities, similar to what is often done in the singlet case. ${ }^{50}$ Such a transformation reduces the cost to $\frac{3}{8} \mathrm{O}^{2} \mathrm{~N}^{4}$.

The $\mathrm{E}$ term is calculated as

$$
\rho_{a i b j}^{E}=\sum_{m n} M_{m i n j} g_{m a n b}
$$

using $g_{\text {manb }}$ from the disk. The terms in square brackets in Eqs. (45)(47) are the $C$ and $D$ intermediates of Ref. 15, which are already available on the disk. All remaining terms cost at most $N^{5}$ and are thus not performance critical.

\section{REFERENCES}

${ }^{1}$ C. Milne, T. Penfold, and M. Chergui, Coord. Chem. Rev. 277-278, 44 (2014), following Chemical Structures using Synchrotron Radiation.

${ }^{2}$ M. Chergui and E. Collet, Chem. Rev. 117, 11025 (2017).

${ }^{3}$ X-Ray Free Electron Lasers: Applications in Materials, Chemistry and Biology, Energy and Environment Series Vol. 18, edited by U. Bergmann, V. Yachandra, and J. Yano (Royal Society of Chemistry, 2017).
${ }^{4}$ P. M. Kraus, M. Zürch, S. K. Cushing, D. M. Neumark, and S. R. Leone, Nat. Rev. Chem. 2, 82 (2018).

${ }^{\mathbf{5}}$ T. Wolf, R. Myhre, J. Cryan, S. Coriani, R. Squibb, A. Battistoni, N. Berrah, C. Bostedt, P. Bucksbaum, G. Coslovich, R. Feifel, K. Gaffney, J. Grilj, T. Martinez, S. Miyabe, S. Moeller, M. Mucke, A. Natan, R. Obaid, T. Osipov, O. Plekan, S. Wang, H. Koch, and M. Gühr, Nat. Commun. 8, 29 (2017).

${ }^{6}$ A. Bhattacherjee, C. D. Pemmaraju, K. Schnorr, A. R. Attar, and S. R. Leone, J. Am. Chem. Soc. 139, 16576 (2017).

${ }^{7}$ P. Norman and A. Dreuw, Chem. Rev. 118, 7208 (2018).

${ }^{8}$ S. P. Neville, V. Averbukh, S. Patchkovskii, M. Ruberti, R. Yun, M. Chergui, A. Stolow, and M. S. Schuurman, Faraday Discuss. 194, 117 (2016).

${ }^{9}$ C. Ehlert, M. Gühr, and P. Saalfrank, J. Chem. Phys. 149, 144112 (2018).

${ }^{10}$ Z. Li, M. E.-A. Madjet, O. Vendrell, and R. Santra, Faraday Discuss. 171, 457 (2014).

${ }^{11}$ M. L. Vidal, X. Feng, E. Epifanovsky, A. I. Krylov, and S. Coriani, J. Chem. Theory Comput. 15, 3117 (2019).

${ }^{12}$ R. Costantini, R. Faber, A. Cossaro, L. Floreano, A. Verdini, C. Hättig, A. Morgante, S. Coriani, and M. Dell'Angela, Commun. Phys. 2, 56 (2019).

${ }^{13}$ S. Coriani and H. Koch, J. Chem. Phys. 143, 181103 (2015).

${ }^{14}$ S. Coriani and H. Koch, J. Chem. Phys. 145, 149901 (2016).

${ }^{15}$ O. Christiansen, H. Koch, A. Halkier, P. Jørgensen, T. Helgaker, and A. Sánchez de Merás, J. Chem. Phys. 105, 6921 (1996).

${ }^{16}$ K. Hald, C. Hättig, and P. Jørgensen, J. Chem. Phys. 113, 7765 (2000).

${ }^{17}$ J. F. Stanton and R. J. Bartlett, J. Chem. Phys. 98, 7029 (1993).

${ }^{18}$ Y. Shao, Z. Gan, E. Epifanovsky, A. T. Gilbert, M. Wormit, J. Kussmann, A. W. Lange, A. Behn, J. Deng, X. Feng, D. Ghosh, M. Goldey, P. R. Horn, L. D. Jacobson, I. Kaliman, R. Z. Khaliullin, T. Kuś, A. Landau, J. Liu, E. I. Proynov, Y. M. Rhee, R. M. Richard, M. A. Rohrdanz, R. P. Steele, E. J. Sundstrom, H. L. Woodcock III, P. M. Zimmerman, D. Zuev, B. Albrecht, E. Alguire, B. Austin, G. J. O. Beran, Y. A. Bernard, E. Berquist, K. Brandhorst, K. B. Bravaya, S. T. Brown, D. Casanova, C.-M. Chang, Y. Chen, S. H. Chien, K. D. Closser, D. L. Crittenden, M. Diedenhofen, R. A. DiStasio, Jr., H. Do, A. D. Dutoi, R. G. Edgar, S. Fatehi, L. Fusti-Molnar, A. Ghysels, A. Golubeva-Zadorozhnaya, J. Gomes, M. W. Hanson-Heine, P. H. Harbach, A. W. Hauser, E. G. Hohenstein, Z. C. Holden, T.-C. Jagau, H. Ji, B. Kaduk, K. Khistyaev, J. Kim, J. Kim, R. A. King, P. Klunzinger, D. Kosenkov, T. Kowalczyk, C. M. Krauter, K. U. Lao, A. D. Laurent, K. V. Lawler, S. V. Levchenko, C. Y. Lin, F. Liu, E. Livshits, R. C. Lochan, A. Luenser, P. Manohar, S. F. Manzer, S.-P. Mao, N. Mardirossian, A. V. Marenich, S. A. Maurer, N. J. Mayhall, E. Neuscamman, C. M. Oana, R. Olivares-Amaya, D. P. O’Neill, J. A. Parkhill, T. M. Perrine, R. Peverati, A. Prociuk, D. R. Rehn, E. Rosta, N. J. Russ, S. M. Sharada, S. Sharma, D. W. Small, A. Sodt, T. Stein, D. Stück, Y.-C. Su, A. J. Thom, T. Tsuchimochi, V. Vanovschi, L. Vogt, O. Vydrov, T. Wang, M. A. Watson, J. Wenzel, A. White, C. F. Williams, J. Yang, S. Yeganeh, S. R. Yost, Z.-Q. You, I. Y. Zhang, X. Zhang, Y. Zhao, B. R. Brooks, G. K. Chan, D. M. Chipman, C. J. Cramer, W. A. Goddard, M. S. Gordon, W. J. Hehre, A. Klamt, H. F. Schaefer III, M. W. Schmidt, C. D. Sherrill, D. G. Truhlar, A. Warshel, X. Xu, A. Aspuru-Guzik, R. Baer, A. T. Bell, N. A. Besley, J.-D. Chai, A. Dreuw, B. D. Dunietz, T. R. Furlani, S. R. Gwaltney, C.-P. Hsu, Y. Jung, J. Kong, D. S. Lambrecht, W. Liang, C. Ochsenfeld, V. A. Rassolov, L. V. Slipchenko, J. E. Subotnik, T. V. Voorhis, J. M. Herbert, A. I. Krylov, P. M. Gill, and M. Head-Gordon, Mol. Phys. 113, 184 (2015).

${ }^{19}$ R. M. Parrish, L. A. Burns, D. G. A. Smith, A. C. Simmonett, A. E. DePrince, E. G. Hohenstein, U. Bozkaya, A. Y. Sokolov, R. Di Remigio, R. M. Richard, J. F. Gonthier, A. M. James, H. R. McAlexander, A. Kumar, M. Saitow, X. Wang, B. P. Pritchard, P. Verma, H. F. Schaefer, K. Patkowski, R. A. King, E. F. Valeev, F. A. Evangelista, J. M. Turney, T. D. Crawford, and C. D. Sherrill, J. Chem. Theory Comput. 13, 3185 (2017).

${ }^{20}$ K. Aidas, C. Angeli, K. L. Bak, V. Bakken, R. Bast, L. Boman, O. Christiansen, R. Cimiraglia, S. Coriani, P. Dahle, E. K. Dalskov, U. Ekström, T. Enevoldsen, J. J. Eriksen, P. Ettenhuber, B. Fernández, L. Ferrighi, H. Fliegl, L. Frediani, K. Hald, A. Halkier, C. Hättig, H. Heiberg, T. Helgaker, A. C. Hennum, H. Hettema, E. Hjertenæs, S. Høst, I.-M. Høyvik, M. F. Iozzi, B. Jansik, H. J. A. Jensen, D. Jonsson, P. Jørgensen, J. Kauczor, S. Kirpekar, T. Kjærgaard, W. Klopper, S. Knecht, R. Kobayashi, H. Koch, J. Kongsted, A. Krapp, K. Kristensen, A. Ligabue, O. B. Lutnæs, J. I. Melo, K. V. Mikkelsen, R. H. Myhre, C. Neiss, 
C. B. Nielsen, P. Norman, J. Olsen, J. M. H. Olsen, A. Osted, M. J. Packer, F. Pawlowski, T. B. Pedersen, P. F. Provasi, S. Reine, Z. Rinkevicius, T. A. Ruden, K. Ruud, V. V. Rybkin, P. Salek, C. C. M. Samson, A. S. de Merás, T. Saue, S. P. A. Sauer, B. Schimmelpfennig, K. Sneskov, A. H. Steindal, K. O. Sylvester-Hvid, P. R. Taylor, A. M. Teale, E. I. Tellgren, D. P. Tew, A. J. Thorvaldsen, L. Thøgersen, O. Vahtras, M. A. Watson, D. J. D. Wilson, M. Ziolkowski, and H. Ågren, Wiley Interdiscip. Rev.: Comput. Mol. Sci. 4, 269 (2014).

${ }^{21}$ F. Furche, R. Ahlrichs, C. Hättig, W. Klopper, M. Sierka, and F. Weigend, Wiley Interdiscip. Rev.: Comput. Mol. Sci. 4, 91 (2014).

${ }^{22}$ J. F. Stanton, J. Gauss, L. Cheng, M. E. Harding, D. A. Matthews, and P. G. Szalay, "CFOUR, coupled-cluster techniques for computational chemistry, a quantum-chemical program package," With contributions from A. A. Auer, R. J. Bartlett, U. Benedikt, C. Berger, D. E. Bernholdt, Y. J. Bomble, O. Christiansen, F. Engel, R. Faber, M. Heckert, O. Heun, M. Hilgenberg, C. Huber, T.-C. Jagau, D. Jonsson, J. Jusélius, T. Kirsch, K. Klein, W. J. Lauderdale, F. Lipparini, T. Metzroth, L. A. Mück, D. P. O’Neill, D. R. Price, E. Prochnow, C. Puzzarini, K. Ruud, F. Schiffmann, W. Schwalbach, C. Simmons, S. Stopkowicz, A. Tajti, J. Vázquez, F. Wang, J. D. Watts and the integral packages MOLECULE (J. Almlöf and P. R. Taylor), PROPS (P. R. Taylor), ABACUS (T. Helgaker, H. J. A. Jensen, P. Jørgensen, and J. Olsen), and ECP routines by A. V. Mitin and C. van Wüllen, For the current version, see http://www.cfour.de.

${ }^{23}$ E. Epifanovsky, M. Wormit, T. Kuś, A. Landau, D. Zuev, K. Khistyaev, P. U. Manohar, I. Kaliman, A. Dreuw, and A. I. Krylov, J. Comput. Chem. 34, 2293 (2013).

${ }^{24}$ C. Hättig, A. Köhn, and K. Hald, J. Chem. Phys. 116, 5401 (2002).

${ }^{25}$ M. Pabst and A. Köhn, J. Chem. Phys. 129, 214101 (2008).

${ }^{26}$ R. J. Squibb, M. Sapunar, A. Ponzi, R. Richter, A. Kivimäki, O. Plekan, P. Finetti, N. Sisourat, V. Zhaunerchyk, T. Marchenko, L. Journel, R. Guillemin, R. Cucini, M. Coreno, C. Grazioli, M. Di Fraia, C. Callegari, K. C. Prince, P. Decleva, M. Simon, J. H. D. Eland, N. Doślić, R. Feifel, and M. N. Piancastelli, Nat. Commun. 9, 63 (2018).

${ }^{27}$ T. Helgaker, P. Jørgensen, and J. Olsen, Molecular Electronic Structure Theory (Wiley, 2004).

${ }^{28}$ H. Koch and P. Jørgensen, J. Chem. Phys. 93, 3333 (1990).

${ }^{29}$ A. I. Krylov, Annu. Rev. Phys. Chem. 59, 433 (2008).

${ }^{30}$ S. Coriani, F. Pawlowski, J. Olsen, and P. Jørgensen, J. Chem. Phys. 144, 024102 (2016).
${ }^{31}$ F. Pawlowski, J. Olsen, and P. Jørgensen, J. Chem. Phys. 142, 114109 (2015).

${ }^{32}$ K. Sneskov and O. Christiansen, Wiley Interdiscip. Rev.: Comput. Mol. Sci. 2, 566 (2012).

${ }^{33}$ R. J. Bartlett, Wiley Interdiscip. Rev.: Comput. Mol. Sci. 2, 126 (2012).

${ }^{34}$ R. Faber and S. Coriani, J. Chem. Theory Comput. 15, 520 (2019).

${ }^{35}$ H. Koch, R. Kobayashi, A. Sanchez de Merás, and P. Jørgensen, J. Chem. Phys. 100, 4393 (1994).

${ }^{36}$ A. Halkier, H. Koch, O. Christiansen, P. Jørgensen, and T. Helgaker, J. Chem. Phys. 107, 849 (1997).

${ }^{37}$ C. Hättig, "Restructuring of the jacobian left transformation in Dalton," private communication (1998).

${ }^{38}$ S. Tsuru, M. L. Vidal, M. Pápai, A. I. Krylov, K. B. Møller, and S. Coriani, J. Chem. Phys. 151, 124114 (2019).

${ }^{39}$ M. L. Vidal, A. I. Krylov, and S. Coriani, "Dyson orbitals within the fc-CVSEOM-CCSD framework: Theory and application to X-ray photoelectron spectroscopy of ground and excited states," Phys. Chem. Chem. Phys. (submitted); e-print ChemRxiv:9616991.v1.

${ }^{40}$ J. P. Carbone, L. Cheng, R. H. Myhre, D. Matthews, H. Koch, and S. Coriani, Adv. Quantum Chem. (in press).

${ }^{41}$ J. Wenzel, M. Wormit, and A. Dreuw, J. Comput. Chem. 35, 1900 (2014).

${ }^{42}$ J. Wenzel, A. Holzer, M. Wormit, and A. Dreuw, J. Chem. Phys. 142, 214104 (2015).

${ }^{43}$ A. Luzanov, A. Sukhorukov, and V. Umanskii, Theor. Exp. Chem. 10, 354 (1976).

${ }^{44}$ G. Schaftenaar and J. Noordik, J. Comput.-Aided Mol. Des. 14, 123 (2000).

${ }^{45}$ E. F. Kjønstad, R. H. Myhre, T. J. Martínez, and H. Koch, J. Chem. Phys. 147, 164105 (2017).

${ }^{46}$ E. F. Kjønstad and H. Koch, J. Phys. Chem. Lett. 8, 4801 (2017).

${ }^{47}$ H. Koch, O. Christiansen, P. Jørgensen, A. M. Sanchez de Merás, and T. Helgaker, J. Chem. Phys. 106, 1808 (1997).

${ }^{48}$ R. Lessard, J. Cuny, G. Cooper, and A. P. Hitchcock, Chem. Phys. 331, 289 (2007).

${ }^{49} \mathrm{C}$. Amis and A. Hitchcock, "Gas phase core excitation database," http:// unicorn.chemistry.mcmaster.ca/corex/cedb-title.html, 2003.

${ }^{50}$ H. Koch, A. Sánchez de Merás, T. Helgaker, and O. Christiansen, J. Chem. Phys. 104, 4157 (1996). 